\title{
Confidence-based Adaptive Extreme Response Surface for Time-Variant Reliability Analysis under Random Excitation
}

\author{
Zequn Wang ${ }^{1}$ and Wei Chen ${ }^{2}$ \\ ${ }^{1}$ Department of Mechanical Engineering-Engineering Mechanics, Michigan Technological \\ University, Houghton, MI 49931, USA \\ ${ }^{2}$ Department of Mechanical Engineering, Northwestern University, Evanston, IL 60208,
} $U S A$

\begin{abstract}
Time-variant reliability analysis aims at revealing the time evolution of the reliability of an engineered system under time-dependent uncertainties that are best described by random processes. In practice, it is still a grand challenge to handle random process in time-variant reliability analysis due to the extremely high computational cost. In this work, a new adaptive extreme response surface (AERS) approach is proposed for time-variant reliability problems. With AERS, the dimensionality of a random process is first reduced to a set of standard normal variables and corresponding deterministic orthogonal functions based on spectral decomposition. As a result, the limit state function is reformulated as a function of only random variables and time. Next, Gaussian process (GP) models are constructed as surrogate models for predicting the value of limit state function at all discretized time nodes to approximate the extreme response surface. The accuracy of GP surrogate models is quantified by a confidence level measure and continuously improved through the sequential adaptive sampling. Using the GP surrogate models, time-dependent reliability is computed via Monte Carlo simulations (MCS). Two case studies are used to demonstrate the effectiveness of the AERS method for time-variant reliability analysis.
\end{abstract}

Key words: time-variant reliability, Gaussian process, spectral decomposition, adaptive response surface, Monte Carlo

\footnotetext{
${ }^{1}$ Assistant Professor, zequnw@mtu.edu

${ }^{2}$ Professor, weichen@ northwestern.edu , Corresponding Author
} 


\section{NOMENCLATURE}

$\begin{array}{lll}R & = & \text { Reliability } \\ \Phi & = & \text { standard Gaussian cumulative distribution function } \\ \beta_{\mathrm{t}} & = & \text { target reliability index } \\ E I & = & \text { expected improvement } \\ f_{x}(x) & = & \text { probability density function } \\ f(\bullet \bullet) & = & \text { conditional probability density function or likelihood function } \\ p_{f} & = & \text { probability of failure } \\ I_{f} & = & \text { indicator function } \\ P_{f} & = & \text { probability of failure } \\ v^{+} & = & \text {out-crossing rate } \\ \lambda(t) & = & \text { out-crossing rate } \\ \mathbf{R} & = & \text { correlation matrix } \\ \mathbf{C o v} & = & \text { covariance matrix } \\ \mathbf{M} & = & \text { GP model } \\ T_{f} & = & \text { time to failure }\end{array}$

\section{INTRODUCTION}

In the past decade, various numerical methods have been developed for reliability problems without considering "time" parameter. Examples of these methods include the most probable point (MPP) based methods [1, 2], the dimension reduction method (DRM) [3-5], the polynomial chaos expansion (PCE) [6-8] and the metamodeling-based methods [9-11]. In such problems, the limit state function, which indicates the state of success or failure, is a time-independent function. However, reliability generally degrades over time due to stochastic operating conditions as well as component deterioration in practical engineering applications. To model the degradation effect, the time parameter has been introduced into the limit state function either directly as an input parameter or indirectly as random process parameters. For instance, the stochastic operating load 
can be characterized as a random process with an explicit time parameter. Even though significant progresses have been made in time-independent cases, ensuring a high level of timevariant reliability during a product life cycle remains challenging in practical engineering applications, as it requires efficient ways for handling time-dependent reliability assessments.

In time-variant reliability analysis, uncertainties are propagated to the response of engineered system to quantitatively assess the probability that the system fulfills its intended function over a specified time period. Uncertainties may come from manufacturing processes, material properties, and operation conditions that can be characterized by random variables and random processes. As a result, the limit state function of system performance is a complex random process such that accurately assessing the time-variant reliability function using simulation-based methods, e.g., Monte Carlo simulation, $[12,13]$ is computationally prohibitive.

Various time-variant reliability methods were developed mainly including the extreme value based approaches [14-18] and the first-passage based approaches [19-23]. The key to extreme performance approaches is to identify the extreme value of a limit state function, and then quantify the uncertainty of the extreme values to approximate time-variant reliability. One example is the nested extreme response surface (NERS) framework [16] which was developed to construct a response surface over the random input space for predicting the time that leads to the extreme response of interest. With the time response surface, time-variant reliability problems are converted to time-independent counterparts, which enables the integration of existing advanced static reliability tools into time-variant reliability approximation. Though NERS shows high efficiency, it lacks the ability of handling random process parameters. In order to tackle random processes as inputs, Mourelatos[18] decomposed the random processes using Eigen analysis and computed the conditional reliability for subsets by the first-order reliability method 
(FORM). The time-variant probability was approximated by the integration of the conditional probabilities over all subsets. The integration of conditional probabilities, however, is computationally expensive requiring numerous FORM calculations and may induce errors for highly nonlinear cases.

The first-passage based approaches were developed based on the "out-crossing events" during a specific time period, a concept first introduced by Rice [23]. A failure occurs if the performance function exceeds the upper bound or falls below the lower bound of the safety threshold. By assuming the independency of out-crossing events, the time-variant reliability is assessed by the integration of the out-crossing rate that represents the rate of reliability change with respect to time. To obtain the out-crossing rate, the PHI2 approach [22] was developed to track the rate in the U-space using FORM, and then to approximate the out-crossing rates based on the reliability indices of two successive time nodes. Although the FORM is seamlessly integrated, the accuracy of out-crossing rate based methods is limited. In addition, the FORM method generally requires accurate sensitivity information of limit state function with respect to random variables, which is often not available in practical engineering applications. Recently, surrogate model-based methods [24-28] have been developed to alleviate the computational burden of time-dependent reliability assessment. Most of existing studies [26, 27] do not incorporate the characteristics of random system variables into adaptive updating scheme, which may constraint the rapid convergence of probability approximations. Another limitation shared by these methods lies in the lack of capability to quantitatively measure the fidelity of surrogate models and the accuracy level of reliability approximations. With existing methods, it is difficult to develop appropriate stopping rules for Kriging updating process. In the literature, a number of studies [29-32] have been done to adaptive update surrogate models based on prior information. 
The efficient global optimization [29] proposed the expected improvement measure to locate new point for updating Kriging models, which shows promising results in deterministic problems. For probability analysis methods [30-32] based on adaptive surrogate models, it still remains challenging to quantitatively measure and effectively enhance the fidelity of surrogate models.

In this work, we propose a time-variant reliability method using adaptive extreme response surfaces that can handle both random variables and random processes as input uncertainty. Using spectral decomposition, random processes are first discretized and represented by a set of independent standard normal variables and deterministic functions. Gaussian process models are then used to build response surfaces for predicting the limit state function at all discretized time nodes. A confidence level is employed to measure the fidelity of GP models and an adaptive sampling scheme is utilized to locate the sample points for updating the GP models. The extreme value of time-dependent function for a given time period is thus approximated by the GP models for instantaneous time nodes within a specified time interval, and MCS are used to approximate the time-variant reliability. AERS possesses several advantages over traditional time-variant reliability methods. First, AERS is a sensitivity-free method, which can reduce the computational cost as well as enhance the robustness of time-variant reliability analysis. Secondly, we can assess the accuracy of time-variant reliability approximation by a quantitative confidence-based measure, which indicates whether an extreme response surface needs further updating. An adaptive updating scheme is used to wisely allocate the computational resource by selecting the most important samples for updating the response surface models.

The paper is arranged as follows. Section 2 introduces existing methods for time-variant reliability analysis. Section 3 describes the proposed adaptive extreme response surface approach including the treatment of input random processes by spectral decomposition, Gaussian 
processing modeling, and the confidence-based adaptive sampling technique. Two case studies are used to demonstrate the effectiveness of the developed methodology in Section 4.

\section{REVIEW OF TIME-VARIANT RELIABILITY ANALYSIS}

In this section, we provide a general definition of time-variant reliability and conduct a literature review of existing methodologies.

\subsection{Definition of Time-Variant Reliability}

In engineering applications, system or component failure occurs if a time-dependent performance function goes beyond its failure threshold. Due to the existence of uncertainties such as those coming from material properties, manufacturing variations, and loading conditions, the preformation function of an engineered system is often a complex stochastic process. With the uncertainties characterized by a set of random variables and random processes, a general limit state function is expressed as $\mathrm{g}(\mathbf{X}, \mathbf{Y}(t), t)$, where $\mathbf{X}=\left[\mathrm{X}_{1}, \mathrm{X}_{2}, \ldots, \mathrm{X}_{r}\right]$ is a vector of $r$ random variables, $\mathbf{Y}(t)=\left[\mathrm{Y}_{1}(t), \mathrm{Y}_{2}(t), \ldots, \mathrm{Y}_{m}(t)\right]$ is a vector of $m$ input random processes with respect to time $t$. Within a specified time period $\left[0, T_{L}\right]$, a failure occurs for a specified realization of $(\mathbf{X}$, $\mathbf{Y}(t))$ if there is a time node $t$ in $\left[0, T_{L}\right]$ such that the limit state value goes beyond the safety margin, which is described by

$$
\exists t \in\left[0, T_{L}\right], g(\mathbf{X}, \mathbf{Y}(t), t)>0 .
$$

The time-variant probability of failure over a time period $(0, T) P_{f}(0, T)$, which is generally a monotonic increasing function with time $T$, is defined as

$$
P_{f}(0, T)=P(\exists t \in[0, T], g(\mathbf{X}, \mathbf{Y}(t), t)>0), 0 \leq T \leq T_{L} .
$$

The corresponding time-variant reliability is given by 


$$
R(0, T)=1-P_{f}(0, T), 0 \leq T \leq T_{L}
$$

It is technically challenging to evaluate the time-variant reliability analytically in Eq. (2) due to the time-dependency of failure events and complexity of limit state functions. Simulationbased methods are extremely computational demanding as a huge number of samples are required for evaluating a limit state function.

Various methods have been developed for time-variant reliability assessment to tackle the "time-dependency issue". These methods can be mainly categorized into two types, out-crossing based methods and extreme value-based methods. As shown in Figure 1, a crossing event occurs if the limit state value jumps from a success state to a failure state. The out-crossing based methods generally analyze crossing events and then approximate the crossing rates along the time-dimension for evaluating time-variant reliability. Instead of focusing on the crossing events, the extreme value-based methods investigate the extreme behavior of a limit state function over time. The extreme distribution of the stochastic process, in this case the limit state function $g(\mathbf{X}$, $\mathbf{Y}(t), t)$, is characterized in time-variant reliability assessment. These two approaches are reviewed briefly next.

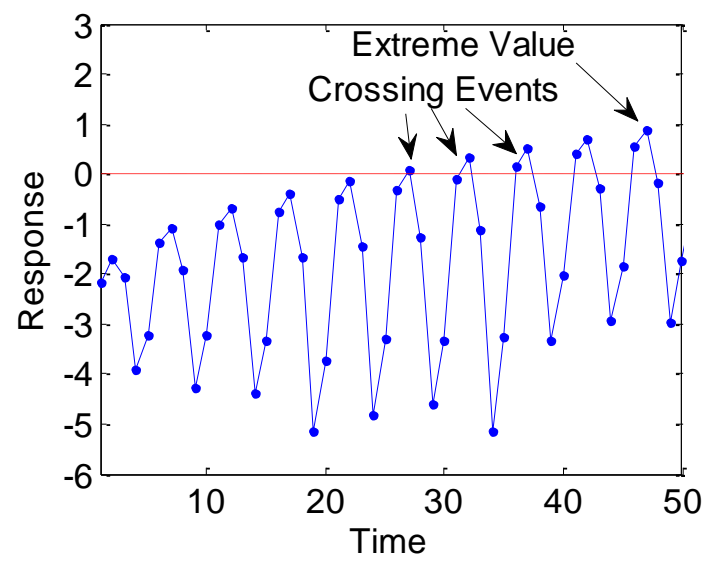

Figure 1: Out-crossing and Extreme Events in Time-variant Reliability 


\subsection{Out-crossing Rate Based Methods}

In the field of time-variant reliability, out-crossing rate based methods were developed based on the concept of failure rate. In engineering applications, the failure rate at time instant $t$ is derived by the limit of a conditional probability as

$$
\lambda(t)=\lim _{\Delta t \rightarrow 0} \frac{\operatorname{Pr}\left(t<T_{f}<t+\Delta t \mid T_{f}>t\right)}{\Delta t},
$$

where $T_{f}$ is the time to failure. With the formulation of the probability of failure in Eq. (2), the failure rate is approximated by the crossing rate as follow

$$
\lambda(t) \approx \lim _{\Delta t \rightarrow 0} \frac{\operatorname{Pr}\{g(\mathbf{X}, \mathbf{Y}(t), t)>0 \bigcap g(\mathbf{X}, \mathbf{Y}(t+\Delta t), t+\Delta t)<0\}}{\Delta t},
$$

with underlying assumptions that all crossing events are independent and the probability of having two or more crossing events within the time period $[t, t+\Delta t]$ is negligible. The timevariant probability of failure is thus calculated as

$$
P_{f}(0, T)=1-\left(1-P_{f}(0,0)\right) \exp \left\{-\int_{0}^{T} \lambda(t) \mathrm{d} t\right\},
$$

where $P_{f}(0,0)$ indicates the instantaneous probability of failure at the initial time $T=0$.

Among the existing studies, the formula developed by Rice [23] has been widely employed for approximating the crossing rate $\lambda(t)$ using the First-Order Reliability Method (FORM) [2]. With FORM, the limit state function $\mathrm{g}(\mathbf{X}, \mathbf{Y}(t), t)$ is transformed into the standard normal space as

$$
g(\mathbf{X}, \mathbf{Y}(t), t)=G\left(\mathbf{U}_{\mathbf{X}}, \mathbf{U}_{\mathbf{Y}}(\mathrm{t})\right),
$$

where $G($.$) represents the corresponding time-dependent limit state function in a standard normal$ space, $\mathbf{U}_{\mathbf{X}}$ and $\mathbf{U}_{\mathbf{Y}}(t)$ are the standard normal random variables transformed from $\mathbf{X}$ and $\mathbf{Y}(t)$. In the standard normal space, FORM iteratively searches the most probable point (MPP), which is 
on the failure surface and has a shortest distance from the origin point. The failure rate is thus approximated [20-23] by the reliability indexes of two successive time nodes obtained by the FORM considering the correlation of the input stochastic process $\mathbf{Y}(t)$.

Even though the out-crossing rate based approaches are mathematically sound, they generally suffer from several limitations. First, the assumption of the independency of out-crossing events may not hold, which may lead to error in approximating time-variant reliability. Secondly, the out-crossing rate based methods may not be accurate and robust, especially for highly nonlinear problems, since the FORM method is based on first-order approximation. In addition, it is technically difficult and computationally expensive to incorporate the out-crossing rate based methods in solving time-variant reliability-based design optimization problems. More information about this approach can be found in references [24-28].

\subsection{Extreme Value Based Methods}

Extreme value based methods focus on the extreme performance value of an engineered system over a specified time period. In the context of time-variant reliability analysis, the extreme value of a limit state function is obtained by maximizing the limit state function within the time period $[0, T]$ if $g(\mathbf{X}, \mathbf{Y}(t), t)>0$ indicates failure, which is described as

$$
g_{e}(\mathbf{X}, T)=\max _{t \in[0, T]} g(\mathbf{X}, \mathbf{Y}(t), t),
$$

where $g_{e}$ is the extreme value of the limit state function for a given realization of the random input $\mathbf{X}$ and random process $\mathbf{Y}(t)$. Apparently, the failure event is exactly determined by the extreme value and the time-variant probability of failure is derived as

$$
P_{f}(0, T)=\operatorname{Pr}\left(g_{e}(\mathbf{X}, T)>0\right) .
$$

Analytically obtaining the probabilistic characteristics of the extreme value $g_{e}$ is usually 
intractable in practice, especially for complex engineering applications while simulation-based methods such as MCS are extremely computationally expensive. In order to overcome the high computational demand, the nested extreme response surface (NERS) method was developed to construct a time response surface that is used to assess an extreme response of the limit state function. In NERS, it is assumed that the limit state function is an explicit function of random variable $\mathbf{X}$ and time $t$, resulting in the elimination of random process $\mathbf{Y}(t)$ in reliability analysis. With this assumption, the response surface of time corresponding to the extreme value of the limit state function, $T_{e}(\mathbf{X})$, is given by

$$
T_{e}(\mathbf{X})=\left\{t \mid \max _{t} g(\mathbf{X}, t), t \in[O, T]\right\} .
$$

Substituting Eq. (10) into Eqs. (8) and (9), the time-variant probability of failure is derived as

$$
P_{f}(0, T)=\operatorname{Pr}\left(g_{e}>0\right)=\operatorname{Pr}\left(g\left(\mathbf{X}, T_{e}(\mathbf{X})\right)>0\right) .
$$

Clearly, time-variant reliability problems are transformed into time-independent counterparts, enabling the use of existing time-independent reliability tools such as the first-order reliability method (FORM), the polynomial chose expansion (PCE), and the dimension reduction method (DRM) for calculating time-variant reliability. The major drawback of the NERS lies in the lack of capability in handling random process parameters. In the next section, we propose the adaptive extreme response surface approach for time-variant reliability assessment that can take into account both random variables and random process parameters.

\section{ADAPTIVE EXTREME RESPONSE SURFACE APPROACH}

This section presents a new time-variant reliability approach, referred to as adaptive extreme response surface (AERS). Section 3.1 presents the spectral decomposition method for modeling the input random process by a set of uncorrelated random variables. The time-dependent extreme 
response function is elaborated in Section 3.2. Section 3.3 describes the Gaussian process modeling technique and the corresponding adaptive sampling technique for enhancing the accuracy of reliability approximations is detailed in Section 3.4. Section 3.5 summarizes the procedure of AERS methodology.

\subsection{Spectral Decomposition of Input Random Processes}

Despite the fact that most of the uncertain quantities appearing in practical engineering problems can be modeled as random parameters (e.g. geometric, material properties), some particular quantities should be treated as random processes such as stochastic loads inserted on structures. In this work, we assume that all input random processes can be modeled as Gaussian stochastic processes that can be fully described by mean function $\mu_{\mathrm{Y}}(t)$, standard deviation function $\sigma_{\mathrm{Y}}(t)$, and autocorrelation function $\rho_{\mathrm{Y}}\left(t_{i}, t_{j}\right)$.

Our goal is to represent a random process $\mathrm{Y}(t)$ by a set of deterministic functions with corresponding random coefficients. At first, the time interval $\left[0, T_{L}\right]$ is discretized using $s$ discrete time nodes $t_{1}=0, \ldots, t s=T_{L}$, and the covariance function between time $t_{i}$ an $t_{j}$ is calculated by $\operatorname{Cov}\left(t_{i}, t_{j}\right)=\sigma_{\mathrm{Y}}\left(t_{i}\right) \sigma_{\mathrm{Y}}\left(t_{j}\right) \rho_{\mathrm{Y}}\left(t_{i}, t_{j}\right)$. The corresponding covariance matrix $\boldsymbol{\Sigma}$ is formed as

$$
\Sigma=\left(\begin{array}{cccc}
\rho\left(t_{1}, t_{1}\right) & \rho\left(t_{1}, t_{2}\right) & \cdots & \rho\left(t_{1}, t_{s}\right) \\
\rho\left(t_{2}, t_{1}\right) & \rho\left(t_{2}, t_{2}\right) & \cdots & \rho\left(t_{2}, t_{s}\right) \\
\vdots & \vdots & \ddots & \vdots \\
\rho\left(t_{s}, t_{1}\right) & \rho\left(t_{s}, t_{1}\right) & \cdots & \rho\left(t_{s}, t_{s}\right)
\end{array}\right) .
$$

By performing eigen decomposition, the covariance matrix $\boldsymbol{\Sigma}$ can be decomposed as $\boldsymbol{\Sigma}=\boldsymbol{\Phi} \cdot \mathbf{I} \bullet \boldsymbol{\Phi}^{\mathbf{T}}$, where $\boldsymbol{\Phi}=\left[\boldsymbol{\Phi}_{1}, \boldsymbol{\Phi}_{2}, \ldots, \boldsymbol{\Phi}_{\mathrm{s}}\right]$ is the matrix of eigenvectors and $\mathbf{I}=\operatorname{diag}\left[\mathrm{I}_{1}, \mathrm{I}_{2}, \ldots, \mathrm{I}_{\mathrm{s}}\right]$ is a diagonal matrix that contains the corresponding eigenvalues. After identifying $p(p<s)$ dominated eigenfunctions, the expansion of the stochastic process $\mathrm{Y}(t)$ is thus given by 


$$
Y(t)=\mu_{Y}(t)+\sum_{i=1}^{p} \sqrt{I_{i}} \cdot \Phi_{i}^{T}(\mathrm{t}) \cdot Z_{i}
$$

where $\mathbf{Z}=\left[Z_{1}, \ldots, Z_{p}\right]$ are uncorrelated standard normal variables. With Eq. (13), the random process $\mathrm{Y}(t)$ is reconstructed by a linear summation of deterministic functions with random weights $\mathbf{Z}$, which transforms the input uncertainties $[\mathbf{X}, \mathbf{Y}(t)]$ to $[\mathbf{X}, \mathbf{Z}]$.

Through the spectral decomposition of stochastic process, the dimensionality of original $s$ correlated random variables in time scale is reduced by an eigen analysis of the covariance matrix without the loss of significant information. The number of dominated eigenfunctions, $p$, should be carefully determined such that the expansion in Eq. (13) is able to capture most of the variation of random process. In this work, $p$ value is determined based on the following criterion [18] as

$$
\sum_{i=1}^{p} I_{i} \cdot \Phi_{i}^{2}(t) / \sum_{i=1}^{s} I_{i} \cdot \Phi_{i}^{2}(t)>\theta, \text { for } t=t_{1}, t_{2}, \ldots, t_{s},
$$

where the critical threshold $\theta$ is set as 0.95 in this study. As a result, the truncated expansion with $p$ terms in Eq. (13) is able to represent at least $95 \%$ variation of the random process $\mathrm{Y}(t)$ over time.

\subsection{Time-dependent Extreme Value Function}

With the employment of spectral decomposition, input random process $\mathrm{Y}(t)$ is represented by a formulation of $p$ random variables $\mathbf{Z}=\left[Z_{1}, Z_{2}, \ldots, Z_{p}\right]$ with the corresponding eigenfunctions. The time-variant limit state function is thus derived as

$$
g(\mathbf{X}, \mathbf{Y}(t), t)=\mathrm{g}_{T}(\mathbf{X}, \mathbf{Z}, t)
$$

If a sample $[x, z]$ is drawn from the joint distribution of random variables $[\mathbf{X}, \mathbf{Z}]$, we then obtain a deterministic realization of random process $g(\mathbf{X}, \mathbf{Y}(t), t)$. The time-dependent extreme value 
function is defined as

$$
g_{e, T}(\mathbf{X}, \mathbf{Z}, T)=\max _{t[0, T]} g_{T}(\mathbf{X}, \mathbf{Z}, t)
$$

where the extreme value $g_{e}($.$) is a function of random variables \mathbf{X}$ and $\mathbf{Z}$, as well as time parameter $T$. Note that the time-dependent extreme value is a monotonic increasing function with time for a realization of random parameters $\mathbf{X}$ and $\mathbf{Z}$. Figure 2 shows a time-dependent extreme value function for a realization of random variables $[\mathrm{X}, \mathrm{Z}]$.

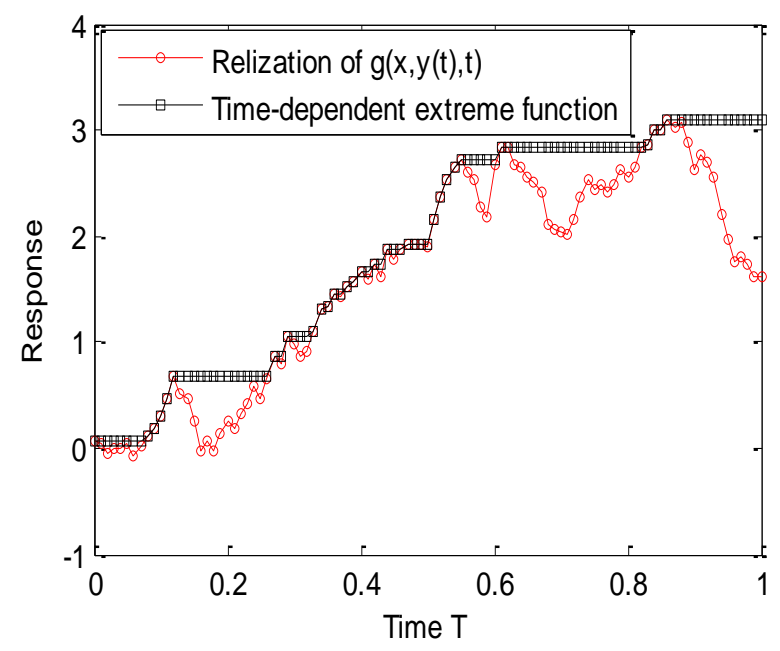

Figure 2: Time-dependent Extreme Value Function

The probability of failure for time interval $[0, T]$ is calculated by

$$
P_{f}(0, T)=\operatorname{Pr}\left(g_{e, T}(\mathbf{X}, \mathbf{Z}, T)>0\right)=\int \cdots \int_{g_{e, T}(\mathbf{X}, \mathbf{Z}, T)>0} f_{\mathbf{X}}(\mathbf{X}) f_{\mathbf{Z}}(\mathbf{Z}) d \mathbf{x} d \mathbf{z}
$$

Since it is unlikely to derive the time-dependent extreme value function and evaluate the multidimension integration analytically in Eq. (17), in this work, a set of GP models will be constructed for predicting the time-dependent extreme value function and approximating the time-dependent probability of failure in Eq. (17) using MCS.

\subsection{Gaussian Process Modeling}


Gaussian process (GP) modeling [33-37] is adopted for building surrogate models in reliability assessment because of its excellent ability of quantifying the surrogate model uncertainty due to the lack of data. The quantification of prediction uncertainty enables the development of an efficient adaptive sampling scheme for updating the surrogate model. In this work, GP models will not be generated for the extreme response surface directly due to its nonsmoothness nature. Instead, a set of GP models is constructed as surrogate models for the transformed limit state function at all time nodes. Let $\mathbf{W}=\left[W_{1}, W_{2}, \ldots, W_{k}\right]$ represents the random input parameters $[\mathbf{X}, \mathbf{Z}]$ for the sake of simplicity, for a given time node $t_{i}(i=1, \ldots, s)$, the limit state function $g_{T}\left(\mathbf{W}, t_{i}\right)$ is generated from the GP model

$$
g_{T}\left(w, t_{i}\right)=\mathbf{f}^{\mathbf{T}}(w) \boldsymbol{\alpha}+S(w),
$$

where $\mathbf{f}^{\mathbf{T}}(w)=\left[f_{1}(w), \ldots, f_{b}(w)\right]$ are basis functions, $\boldsymbol{\alpha}=\left[\alpha_{1}, \ldots, \alpha_{b}\right]$ is a regression coefficient vector, and $S(w)$ is a Gaussian random process with zero mean and a covariance matrix. In this study, a constant mean $\mu$ is used to replace the term $\mathbf{f}^{\mathbf{T}}(w) \boldsymbol{\alpha}$ since a GP model with a constant mean is often adequate for modeling nonlinear responses in engineering applications. The covariance function of stochastic process $S(w)$ between two input variables $w_{i}$ and $w_{j}$ is expressed as

$$
\operatorname{Cov}_{(i, j)}=\sigma^{2} \mathbf{R}_{(i, j)},
$$

where $\mathbf{R}$ represents the correlation matrix. The $(i, j)$ entry of $\mathbf{R}$ is described as

$$
\mathbf{R}_{(i, j)}=\operatorname{Corr}\left(w_{i}, w_{j}\right)=\exp \left[-\sum_{u=1}^{k} a_{u}\left|w_{i, u}-w_{j, u}\right|^{b_{u}}\right],
$$

where Corr is the correlation function, and $a_{u}$ and $b_{u}(u=1, \ldots, k)$ are parameters of the GP model to be determined. With $n$ number of observations $(\mathbf{w}, \mathbf{G})$, the log likelihood function of the GP model parameters can be provided as 


$$
L_{G P}=-\frac{1}{2}\left[n \ln (2 \pi)+n \ln \sigma^{2}+\ln |\mathbf{R}|+\frac{1}{2 \sigma^{2}}(\mathbf{G}-\mathbf{A} \mu)^{T} \mathbf{R}^{-1}(\mathbf{G}-\mathbf{A} \mu)\right]
$$

where $\mathbf{A}$ is an $n \times 1$ unit vector, $\mathbf{G}$ is the observations of training data. The unknown parameters are determined by maximizing the likelihood function in Eq. (21). Once the GP model is constructed based on sampling data, the response for any given new input point $w^{\prime}$ can be estimated as

$$
g_{T}\left(w^{\prime}, t_{i}\right)=\mu+\mathbf{r}^{T} \mathbf{R}^{-1}(\mathbf{G}-\mathbf{A} \mu),
$$

where $\mathbf{r}$ is the correlation vector between $w^{\prime}$ and the samples $\mathbf{w}=\left[w_{1}, \ldots, w_{n}\right]$, of which the $i^{\text {th }}$ element of $\mathbf{r}$ is given by $\mathbf{r}(i)=\operatorname{Corr}\left(w^{\prime}, w_{i}\right)$. The mean square error $e\left(w^{\prime}, t_{i}\right)$ can be estimated by

$$
\hat{e}\left(w^{\prime}, t_{i}\right)=\sigma^{2}\left[1-\mathbf{r}^{T} \mathbf{R}^{-1} \mathbf{r}+\frac{\left(1-\mathbf{A}^{T} \mathbf{R}^{-1} \mathbf{r}\right)^{2}}{\mathbf{A}^{T} \mathbf{R}^{-1} \mathbf{A}}\right] .
$$

\subsection{Confidence-Based Adaptive Sampling}

Using the GP model of the limit state function $\mathrm{g}_{T}\left(\mathbf{W}, t_{i}\right)$, the static probability of failure is obtained by MCS. Let $\Omega=\left\{\mathbf{w}, \mid g_{T}\left(\mathbf{w}, t_{i}\right)>0\right\}$ denotes the failure region, the probability of failure is expressed as

$$
P_{f}\left(t_{i}, t_{i}\right)=\operatorname{Pr}\left(g_{T}\left(\mathbf{W}, t_{i}\right) \in \Omega\right)=\int_{R^{k}} I\left(w, t_{i}\right) f_{w}(w) d w=\mathrm{E}\left[I\left(w, t_{i}\right)\right]
$$

where $\operatorname{Pr}($.$) represents a probability measure; E[.] denotes the expectation operator; R^{k}$ is the real number space; $I\left(w, t_{i}\right)$ is an indicator function approximated by the GP model at the $i^{\text {th }}$ time node and defined as

$$
I\left(w, t_{i}\right)=\left\{\begin{array}{lr}
1, & g_{T}\left(w, t_{i}\right)>0 \\
0, & \text { otherwise }
\end{array},\right.
$$

where $g_{T}\left(w, t_{i}\right)$ is an approximation by Eq. (22) from the GP model. With $N$ randomly generated sample points in MCS, the time-independent reliability at time $t_{i}$ is computed by 


$$
R\left(t_{i}, t_{i}\right)=1-P_{f}\left(t_{i}, t_{i}\right)=1-\frac{1}{N} \sum_{i=1}^{N} I\left(w_{i}, t_{i}\right)
$$

Due to the lack of data in building a GP model, the reliability approximation in Eq. (26) highly depends on the fidelity of the GP model. Thus a quantitative measure is desired to quantify the confidence level of reliability approximation; this measure will guide the adaptive sampling scheme for model updating.

For the $i^{\text {th }}$ MCS sample $\mathbf{W}=\left[w_{1}, . ., w_{N}\right]$, the unknown response of $w_{i}$ is predicted by the GP model as a normal random variable with mean and variance computed by Eqs. (22) and (23) respectively. Thus, $w_{i}$ can be simply classified as failure or success according to Eq. (25) based on the prediction mean. Due to the prediction uncertainty, the probability of correct classification for $w_{i}$ is defined as

$$
\operatorname{Pr}_{c}\left(w_{i}, t_{i}\right)=\Phi\left(\frac{\left|g_{T}\left(w_{i}, t_{i}\right)\right|}{\sqrt{\hat{e}\left(w_{i}, t_{i}\right)}}\right),
$$

where |.| is the absolute operator. In this work, we define the confidence of reliability approximation [9] as the expected value of the probability of correct classification, with respect to the uncertainty in $\mathbf{W}$,

$$
C L\left(t_{i}\right)=\mathrm{E}\left[\mathbf{P r}_{\mathbf{c}}\right]=\frac{1}{N} \sum_{i=1}^{N} \operatorname{Pr}_{c}\left(w_{i}, t_{i}\right)
$$

Note that $C L$ is a positive value within $(0.5,1]$; a large value of $C L$ represents less uncertainty in reliability approximation.

For enhancing the confidence of using the GP model for reliability approximation, an adaptive sampling technique is utilized to identify the most important sample point for updating the GP model in a sequential and iterative procedure. The new sample point is selected within the MCS sample pool $\mathbf{W}=\left[w_{1}, . ., w_{N}\right]$ by calculating the importance measure of sample points, 
which is defined in this work as,

$$
\psi\left(w_{i}, t_{i}\right)=\left(1-\operatorname{Pr}_{c}\left(w_{i}, t_{i}\right)\right) \times f_{w}\left(w_{i}\right) \times \sqrt{\hat{e}\left(w_{i}, t_{i}\right)}
$$

where $f_{w}\left(w_{i}\right)$ is the probability density function value at $w_{i} ; \hat{e}\left(w_{i}, t_{i}\right)$ is the estimated mean square error of the prediction using the GP model. Thus, the most important sample $\left(w^{\prime}, t_{i}\right)$ is identified by maximizing $\psi($.$) as$

$$
\left.w^{\prime}\right|_{t=t_{i}}=\underset{i}{\arg \max } \psi\left(w_{i}, t_{i}\right), i=1, \ldots, N
$$

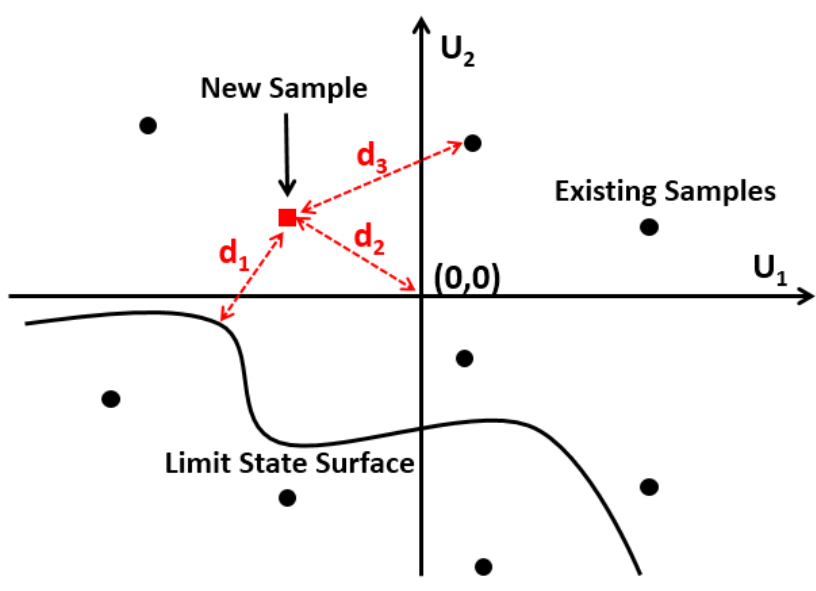

Figure 3: Adaptive Sampling Scheme in U-Space

Figure 3 qualitatively illustrates the confidence-based adaptive sampling strategy in the transformed standard normal space. The first term in Eq. (29) reaches the maximum when the distance $d_{1}$ is zero, which indicates that the sample points on the failure surface may enhance the confidence level of reliability approximation the most. The second term $f w(w)$ can be interpreted by the distance $\mathrm{d}_{2}$ in the standard normal space. Thus multiplication of the first two terms in Eq. (29) leads to a sample point on the limit state surface with the minimum distance to origin. The third term in the importance measure is the predication uncertainty of surrogate models due to lack of data. With the third term in the importance measure, a sample point with larger prediction uncertainty will be more likely to be selected for updating purpose. Maximizing the third term, 
which is prediction uncertainty, will ensure maximizing the distance of the new point from existing training points. By including the prediction variance $\hat{e}\left(w_{i}, t_{i}\right)$ in Eq. (29), the sample points for updating GP models will be sparsely allocated to reduce the surrogate model uncertainty due to the lack of data. As a result, important sample points are identified to adaptively learn the limit state surface.

With the proposed adaptive updating scheme, sample points are obtained at the most critical locations at each time node. All GP models will be updated sequentially till the corresponding confidence levels of instantaneous reliability approximations $C L\left(t_{i}\right)(i=1, \ldots, s)$ by Eq. (28) meets the confidence target. Next for a given time interval $[0, T]$ where $T=t_{q}$, the time-variant probability of failure is approximated by

$$
P_{f}(0, T)=\left.\int_{R^{k}} I(w)\right|_{[0, T]} f_{w}(w) d w=\mathrm{E}\left[\left.I(w)\right|_{[0, T]}\right]
$$

where $\left.I(w)\right|_{[0, T]}$ is an indicator function for extreme response surface approximated by multiple GP models at multiple time points, which is expressed as

$$
\left.I(w)\right|_{[0, T]}=\left\{\begin{array}{lr}
1, & \max _{1 \leq i \leq q} g_{T}\left(w, t_{i}\right)>0 \\
0, & \text { otherwise }
\end{array},\right.
$$

where $g_{T}\left(w, t_{i}\right)(1 \leqslant i \leqslant q)$ are approximated using the GP models $\mathbf{M}_{1} \sim \mathbf{M}_{q}$, With $N$ randomly generated sample points in MCS, the time-independent reliability at time interval $[0, T]$ is computed by

$$
R(0, T)=1-P_{f}(0, T)=1-\left.\frac{1}{N} \sum_{i=1}^{N} I(w)\right|_{[0, T]} .
$$

\subsection{AERS Algorithm Procedure}

The following steps summarize the AERS approach based on the description in Secs. 3.1-3.4.

Step 1: Initialize the life time of interest $T_{L}$, the number of time nodes $s$, the confidence 
target $C L_{T}$, the number of sample points $N$ in MCS, the number of initial sample points $n$ for building initial GP models

Step 2: Decompose the input random processes in the time-dependent limit state function using the spectral decomposition, determine the number of dominated eigenfunctions $p$, and form a set of random input parameters $\mathbf{W}$

Step 3: Generate $n$ sample points according to the randomness of $\mathbf{W}$, and evaluate the time series response of each sample point. Construct $s$ GP models for the limit state function at all time nodes.

Step 4: For each time node, draw the same $N$ sample points from $\mathbf{W}$ for approximating instantaneous probability of failure using MCS while the response of interest is predicted by GP models. The GP models are updated by identifying more sample points till the confidence level of reliability estimation is satisfied.

Step 5: Approximate the extreme performances of all $N$ sample points and classify the states as success or failure based on Eq. (32), and compute time-variant reliability using MCS via Eq.

The flowchart of the adaptive extreme response approach (AERS) is shown in Figure 4. As a simulation-based time-variant reliability approach, AERS builds surrogate models using the Gaussian process modeling technique for the extreme response surface, which is then utilized to compute time-variant reliability in MCS. In spectral decomposition, it is worth noting that the number of transformed random parameters depends on the correlation length of stochastic processes. It could be a large number if the random process tends to more stochastic. For high dimensional sample space, we generally recommend more initial sample points to construct an initial surrogate model and then the adaptive sampling scheme will be triggered to update the 
surrogate model by identifying new importance sample points. For high dimensional problems, the updating process could be expensive as the prediction uncertainty is significant due to lack of data. Although the proposed method is still able to perform reliability analysis for these cases, the high computational cost could be a concern. It is expected that the AERS should outperform the MCS method and the existing analytical methods such as the out-crossing rate based methods in terms of accuracy and efficiency. By introducing surrogate modes in AERS, vast computational efforts can be saved compared with direct MCS, however, the accuracy of reliability approximation is not assured due to the lack of data in surrogate modeling. AERS addresses this issue by employing a confidence level measure that takes into account the uncertainty in GP models. The updating mechanism will be triggered and sequentially repeated if the confidence level target is not satisfied. It is generally recommended to set a confidence level target for reliability analysis if the time-independent probability of failure is small. In terms of efficiency, AERS reduces the computational cost by developing a time-dependent adaptive sampling scheme that can smartly locates the critical time nodes for updating. In principle, the critical time nodes with larger instantaneous probability of failure will be allocated more sample points. In addition, the confidence-based sampling scheme can locate critical sample points for instantaneous time nodes that may have greater potential for improving the confidence level of GP models. Thus, AERS is expected to be as efficient as the analytical time-variant reliability approaches as demonstrated in the case study 1 in Section 4. 


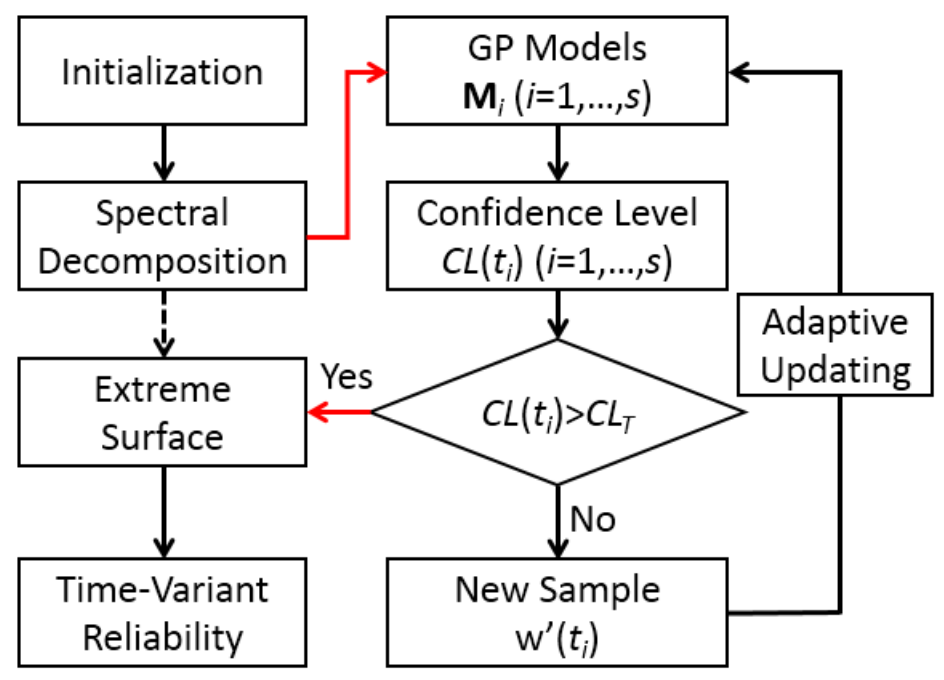

Figure 4: Flowchart of Adaptive Extreme Response Surface Approach

\section{CASE STUDIES}

In this section, two case studies, a mathematical example and a hydrokinetic turbine blade, are used to illustrate the performance of the AERS approach.

\subsection{Case Study I: A Math Example}

A mathematical example is employed to demonstrate the time-variant reliability analysis using the AERS approach. A time-variant limit state function $G(\mathbf{X}, Y(\underline{t}), t)$ is given by

$$
g(\mathbf{X}, Y(t), t)=20-X_{1}^{2} X_{2}+5 X_{1}(1+Y(t)) t-\left(X_{2}+1\right) t^{2}
$$

where $t$ represents the time variable varying within $[0,1] ; \mathbf{X}=\left[\mathrm{X}_{1}, \mathrm{X}_{2}\right]$ are normally distributed random variables, $Y(t)$ is a Gaussian process with zero mean and unit variance while the corresponding correlation function is given by

$$
\rho\left(t_{1}, t_{2}\right)=\exp \left(-\left(t_{2}-t_{1}\right)^{2}\right)
$$

Failure occurs if $g(\mathbf{X}, Y(t), t)$ is greater than zero. Figure 5 shows limit state surfaces at time $t=1$ by fixing $\mathrm{X}_{1}$ and $\mathrm{X}_{2}$ as 3.5 respectively. 

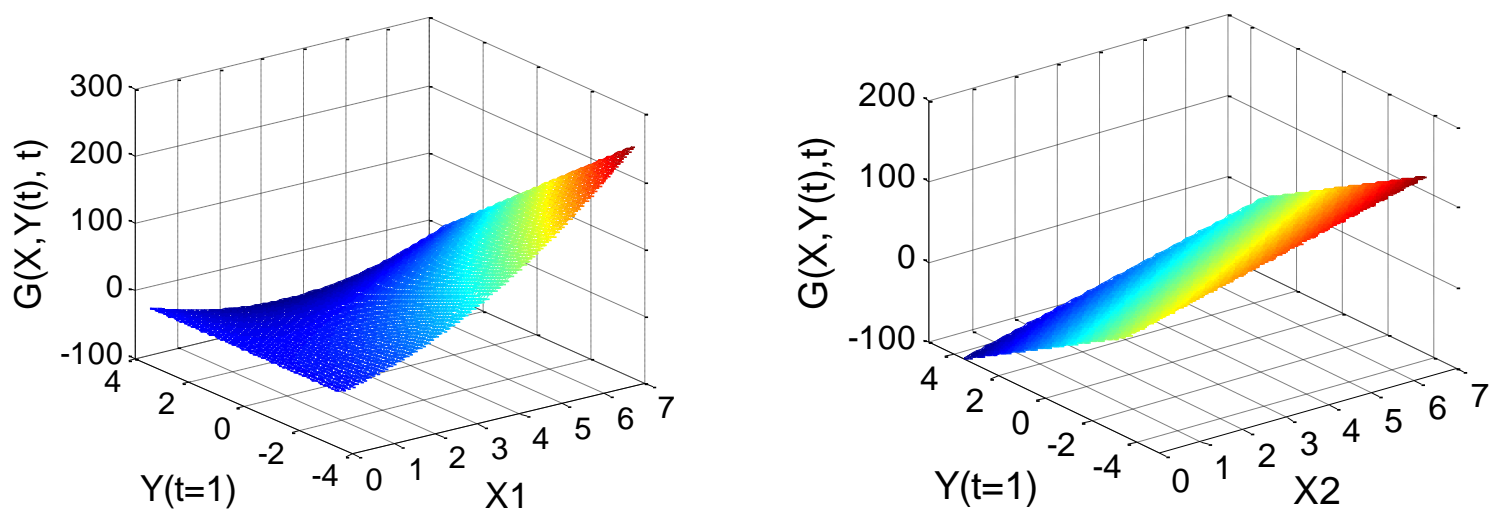

Figure 5: Limit State Surface at Time $t=1$ a) $X_{2}=3.5$; b) $X_{1}=3.5$

The time-variant reliability problem is solved for the system inputs: $X_{1}$ and $X_{2}$ follow a normal distribution $N\left(3.5,0.25^{2}\right)$. As a benchmark solution, we employed the direct MCS to calculate the time-variant reliability with $10^{6}$ random sample points. With MCS, the random process $Y(t)$ is first discretized into 20 time intervals evenly with 21 time nodes. The covariance matrix is thus obtained based on the correlation function given by Eq. (35), and utilized to compute eigenvalues and eigenfunctions.
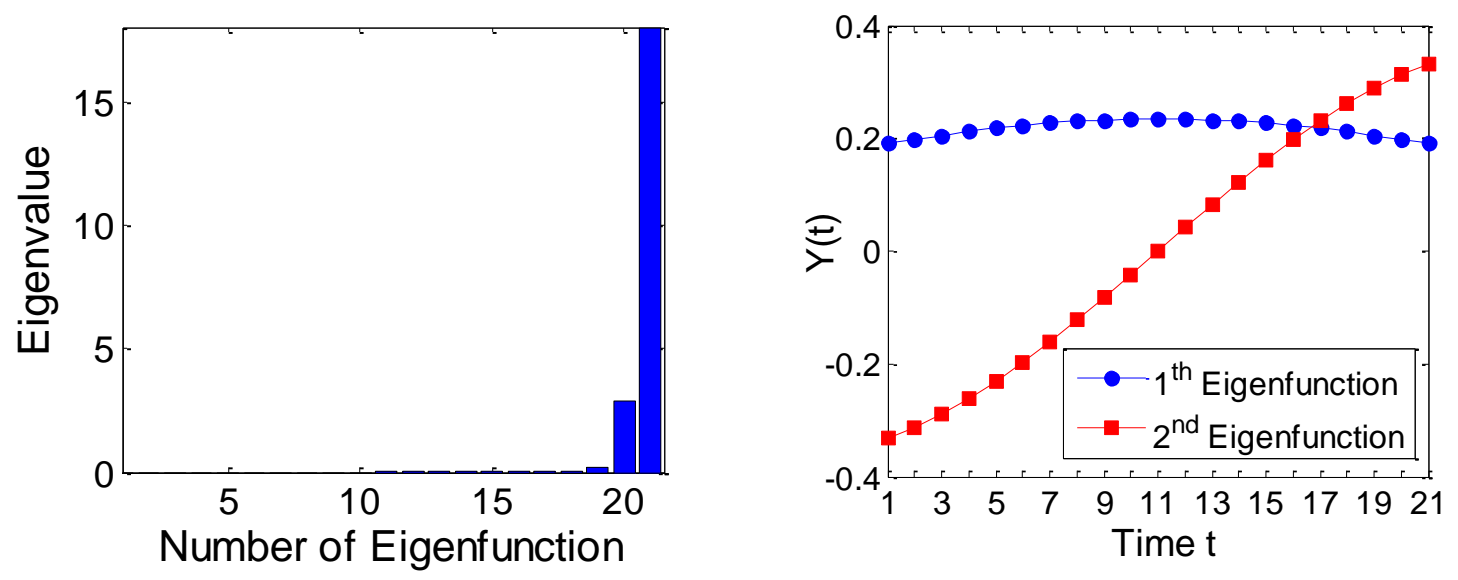

Figure 6: Eigenvalues and Eigenfunctions of $\mathrm{Y}(t)$

In order to cover at least $95 \%$ variation of the random process $Y(t)$, two most important eigenfunctions with the standard normal variables $\mathbf{Z}=\left[\mathrm{Z}_{1}, \mathrm{Z}_{2}\right]$ as coefficients are included in the 
expansion to represent $Y(t)$. Figure 6 demonstrates the vector of eigenvalues and the most two dominated eigenfunctions. With MCS, $10^{6}$ samples are generated for both $\mathbf{X}$ and $\mathbf{Z}$ and the timedependent limit state function is evaluated accordingly.

In what follows, we first carry out the time-variant reliability analysis using the AERS approach and then we compare the AERS results with those from the out-crossing rate-based approach and MCS for the same example to demonstrate its efficacy. Similarly using the MCS method, the AERS approach discretizes the random process $\mathrm{Y}(t)$ by a time interval $\Delta \mathrm{t}=0.05$ and truncates the expansion with two terms. Then the limit state function $\mathrm{g}(\mathbf{X}, Y(t), t)$ is reformulated as $\mathrm{g}_{T}(\mathbf{X}, \mathbf{Z}, t)$ where $\mathbf{X}=\left[\mathrm{X}_{1}, \mathrm{X}_{2}\right]$ and $\mathbf{Z}=\left[\mathrm{Z}_{1}, \mathrm{Z}_{2}\right]$. For each time node $t_{i}(i=1,2, \ldots, 21), 40$ initial sample points are generated for $\mathbf{X}$ and $\mathbf{Z}$ using the Latin hypercube sampling technique to obtain the limit state value $\mathrm{g}_{T}(\mathbf{X}, \mathbf{Z}, t)$, then a GP model $\mathbf{M}_{i}$ is constructed for the limit state function $\mathrm{g}_{T}\left(\mathbf{X}, \mathbf{Z}, t=t_{\mathrm{i}}\right)$. The unkonwn parameters of the GP model are estimated by maximizing the likelihood function.

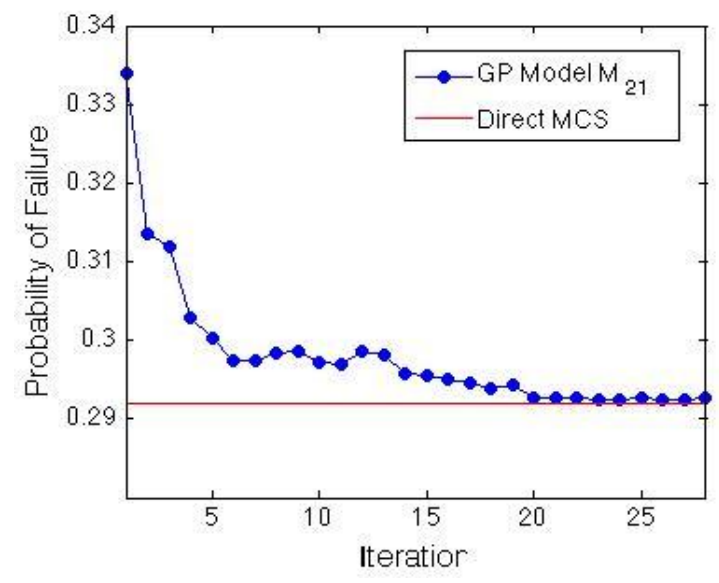

a) Instantaneous Probabilty of Failure

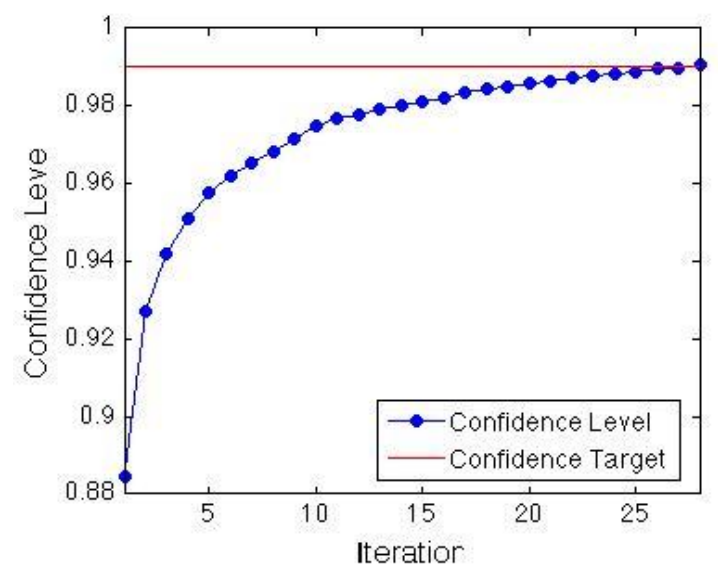

b) Iterative Confidence Level

Figure 7: Updating Process of GP Model $\mathbf{M}_{21}$ for the $21^{\text {th }}$ Time Node

The instantaneous reliability at time node $t_{i}$ is thus computed by MCS in which the limit state value is predicted by the GP model. The confidence level of reliability approximation is 
computed, and then compared with a predefiend confidence target $C L_{\mathrm{T}}$ which is set as 0.99 in this case. The GP model is updated by identifying and evaluting the most useful sample points sequentially until the confidence level is satified. Figure 7 illustrates the updaing process in construing GP model $\mathbf{M}_{21}$ for time node $\mathrm{t}_{21}$.
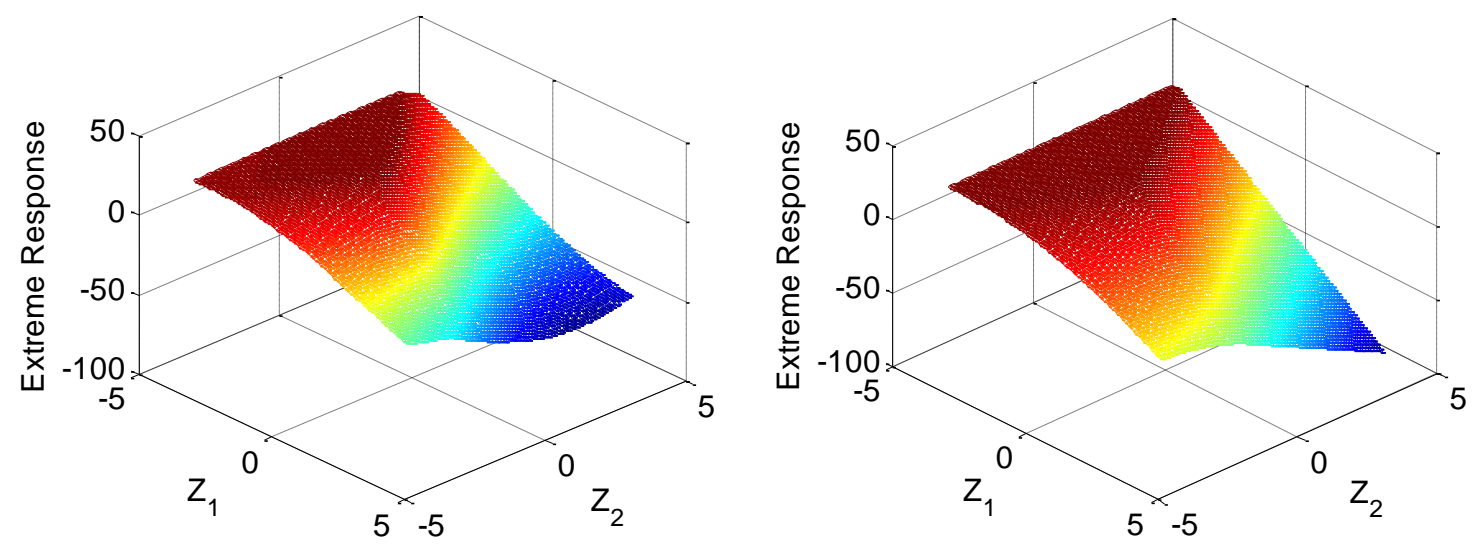

Figure 8: Extreme Response Surface a) AERS b) MCS

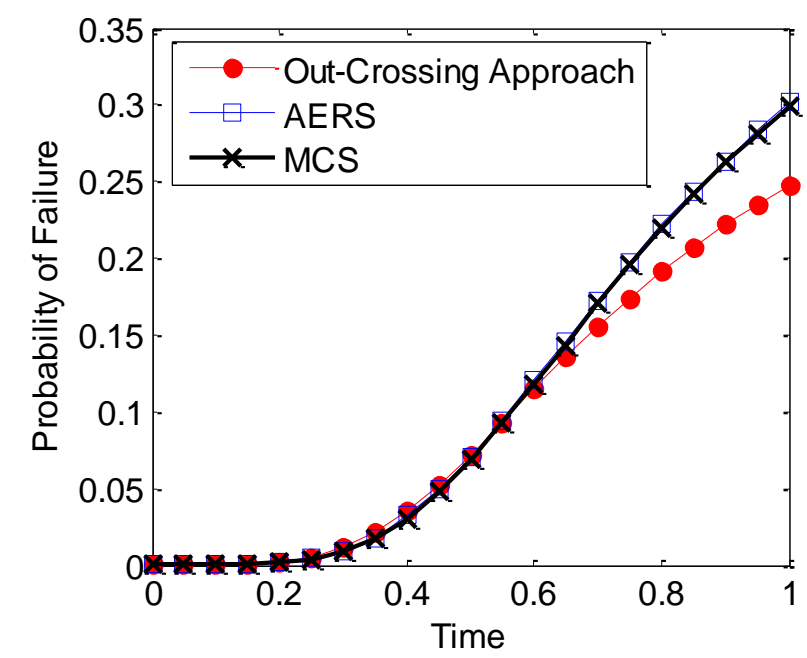

Figure 9: Time-Variant Probability of Failure

Table 1 Time-Variant Probability of Failure

\begin{tabular}{cccc}
\hline Time Interval & MCS & AERS & $\begin{array}{c}\text { 'Out-Crossing } \\
\text { Rate Method }\end{array}$ \\
\hline$[0,0.2]$ & 0.00144 & 0.00169 & 0.00197 \\
{$[0,0.4]$} & 0.03050 & 0.03229 & 0.03511
\end{tabular}




\begin{tabular}{cccc}
{$[0,0.6]$} & 0.11820 & 0.12007 & 0.11458 \\
{$[0,0.8]$} & 0.21952 & 0.22242 & 0.19162 \\
{$[0,1.0]$} & 0.29860 & 0.30133 & 0.24701 \\
\# of Fun & $10^{6}$ & $840+258$ & 1116 \\
\hline
\end{tabular}

Once the GP models are obtained for all time nodes, the extreme response surface for any given time interval is approximated by the corresponding GP models. Figure 8 shows the extreme response surface over the $\mathbf{Z}$ space by fixing $\mathbf{X}$ at its mean value. With the extreme response surface, the AERS approach computes the time-variant probability of failure without extra computational cost. The out-crossing rate-based approach [20] is employed to solve the same problem, in which time is also discretized with 21 time nodes evenly. The results from AERS, out-crossing rate-based approach, and direct MCS are detailed in Figure 9 and Table 1. With AERS, 840 function evaluations are used to construct initial GP models while 258 are required for confidence-based updating. It is observed that the AERS can achieve better accuracy in time-variant reliability assessment while slightly less computational cost is required compared with the out-crossing rate-based approach.

\subsection{Case Study II: Hydrokinetic Turbine Blade}

A hydrokinetic turbine blade [38] is used to demonstrate the developed approach for timedependent reliability analysis. Belong to the category of renewable energy; hydrokinetic turbines convert the kinetic energy of flowing water into mechanical work by utilizing hydrodynamic blades under the time-dependent river flow load. In order to prevent potential failures in operation, achieving high life-cycle reliability is of vital importance in the design process of hydrokinetic turbine blade.

The shape of turbine blades essentially impacts the safety and efficiency of the hydrokinetic turbine system. As shown in Figure 10 the cross section of the blade can be fully described by 
three geometry parameters, $d_{1}, d_{2}$, and $d_{3}$. In order to characterize the time-dependent river flow, the monthly river velocity is modeled by a random process $\mathrm{v}(t)$ with mean $\mu_{\mathrm{v}}(t)$, standard deviation $\sigma_{\mathrm{v}}(t)$, and autocorrelation function $\rho_{\mathrm{v}}\left(t_{1}, t_{2}\right)$ given by

$$
\begin{gathered}
\mu_{v}(t)=\sum_{i=1}^{4} a_{i}^{m} \sin \left(b_{i}^{m} t+c_{i}^{m}\right), \\
\sigma_{v}(t)=\sum_{j=1}^{4} a_{i}^{s} \exp \left\{-\left[\left(t-b_{j}^{s}\right) / c_{j}^{s}\right]\right\}, \\
\rho_{v}\left(t_{1}, t_{2}\right)=\cos \left(2 \pi\left(t_{2}-t_{1}\right)\right),
\end{gathered}
$$

where constants $a b c$ for Eqs. (36) and (37) are

$$
\begin{gathered}
a_{1}^{m}=3.815, a_{2}^{m}=2.528, a_{3}^{m}=1.176, a_{4}^{m}=-0.07856 \\
b_{1}^{m}=0.2895, b_{2}^{m}=0.5887, b_{3}^{m}=0.7619, b_{4}^{m}=2.183, \\
c_{1}^{m}=-0.2668, c_{2}^{m}=0.9651, c_{3}^{m}=3.116, c_{4}^{m}=-3.161, \\
a_{1}^{s}=0.7382, a_{2}^{s}=1.013, a_{3}^{s}=1.875, a_{4}^{s}=1.283, \\
b_{1}^{s}=6.456, b_{2}^{s}=4.075, b_{3}^{s}=9.913, b_{4}^{s}=1.035 \\
c_{1}^{s}=0.9193, c_{2}^{s}=1.561, c_{3}^{s}=6.959, c_{4}^{s}=2.237 .
\end{gathered}
$$

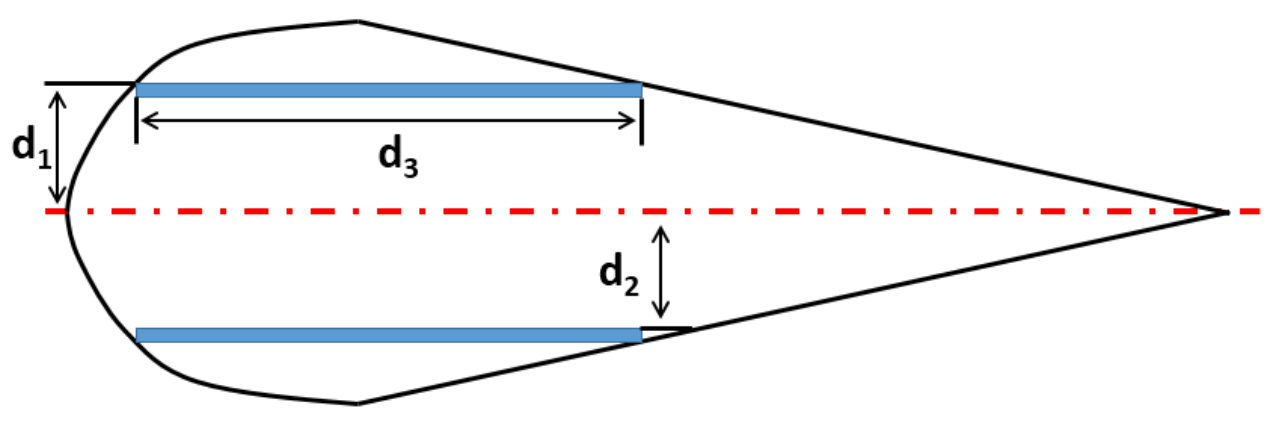

Figure 10: Cross Section of the Tubing Blade

The bending moment $M_{b}$ at the root of the blade under the stochastic load $\mathrm{v}(t)$ is given by 


$$
M_{b}=\frac{1}{2} \rho v^{2}(t) C_{m},
$$

where $\rho=10^{3} \mathrm{~kg} / \mathrm{m}^{3}$ is the water density, $C_{m}=0.3422$ represents the coefficient of moment. The limit state function of hydrokinetic turbine blade is thus defined by

$$
g(\cdot)=\frac{d_{1} M_{b}}{E I_{a}}-A_{l}
$$

where $A_{l}$ is the allowable limit of the material stain, $M_{b}$ is the flapwise bending moment at the root of the blade, $E=14 \mathrm{GPa}$ is the Young's modulus, and $I_{a}$ is the area moment of inertia at the root of the blade, which is given by

$$
I_{a}=\frac{2}{3} d_{3}\left(d_{1}^{3}-d_{2}^{3}\right)
$$

In this study, $d_{1}, d_{2}, d_{3}$ and $A_{l}$ are random variables while all others are deterministic. The distribution information of random parameters is listed in Table 2.

Table 2: Random Variables for Hydrokinetic Turbine Blade

\begin{tabular}{ccccc}
\hline Variables & Distribution & Mean $(\mathrm{mm})$ & Std Dev. $(\mathrm{mm})$ & Auto Cor. \\
\hline$d_{1}$ & Normal & 0.22 & 0.00220 & N/A \\
$d_{2}$ & Normal & 0.025 & 0.00025 & N/A \\
$d_{3}$ & Normal & 0.019 & 0.00019 & N/A \\
$A_{l}$ & Normal & 0.025 & 0.00025 & N/A \\
$v(t)$ & GP & $\mu_{\mathrm{v}}(\mathrm{t})$ & $\sigma_{\mathrm{v}}(\mathrm{t})$ & $\rho_{\mathrm{v}}(\mathrm{t})$ \\
\hline \hline
\end{tabular}

Following the procedure in Section 3.5, the time interval $[0,12]$ months were discretized using a time step 0.2 month. In the spectral decomposition of the random process $\mathrm{v}(t)$, the covariance matrix was formed based on Eqs. (37) and (38). Then eigen analysis was performed to obtain the eigenvalues and corresponding eigenvectors of the covariance matrix. Two important eigenfunctions are identified to represent the random process $\mathrm{v}(t)$. Figure 11 shows ten 
realizations of the random process $\mathrm{v}(t)$ and limit state functions.
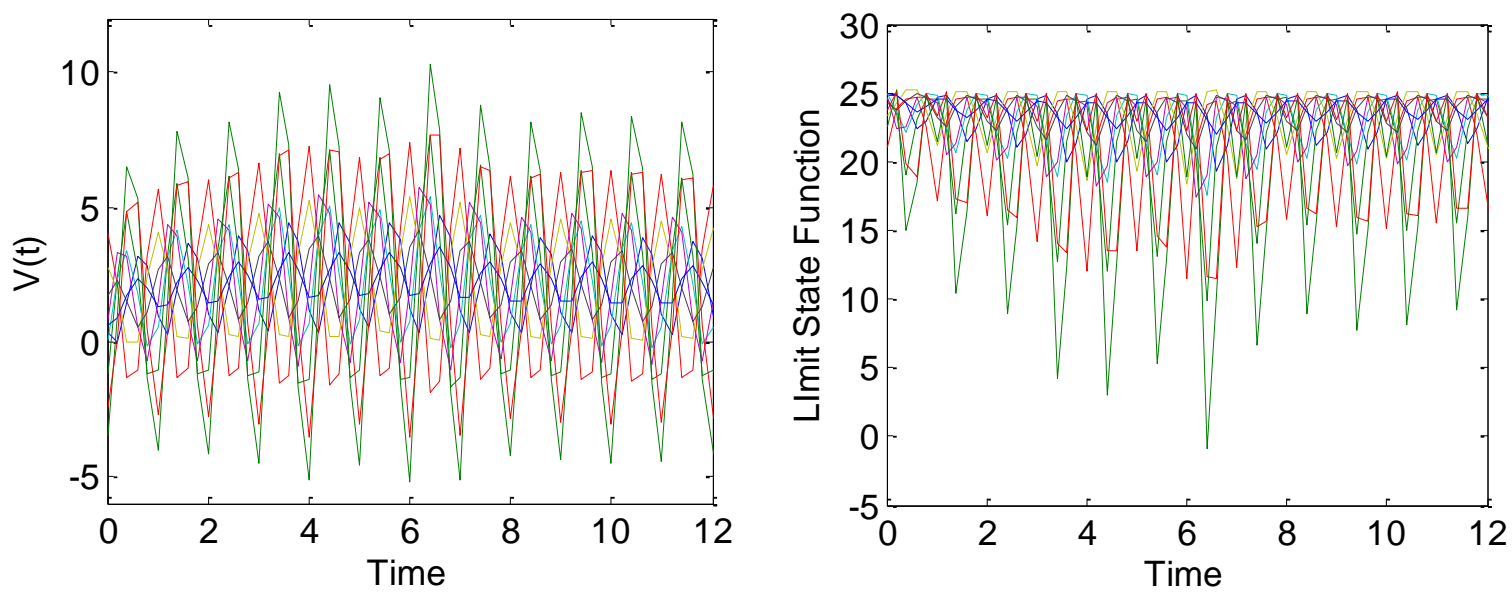

Figure 11: Realizations of Random Process v $(t)$ and Limit State Functions

With two standard normal variables $\mathbf{Z}=\left[\mathrm{Z}_{1}, \mathrm{Z}_{2}\right]$ introduced by the random process $\mathrm{v}(t), 60$ samples are generated for $[\mathbf{X}, \mathbf{Z}]$ and evaluated for the limit state function. For the $i^{\text {th }}$ time node $(i=1,2, . ., 61)$, an initial GP model is constructed for approximating the static reliability. By setting 0.99999 as the confidence target, all GP models are updated adaptively till the confidence requirement is met. It is observed that the time-variant probability of failure is in the some order of magnitude for both case studies II and I. However different confidence level targets are employed mainly because the time-independent probabilities of failure for the second case study is much smaller. The extreme response surface for any given time interval can be approximated by the updated GP models within the particular time period. Figure 12 shows the extreme response surfaces by fixing $\mathbf{X}$ at the mean values. 

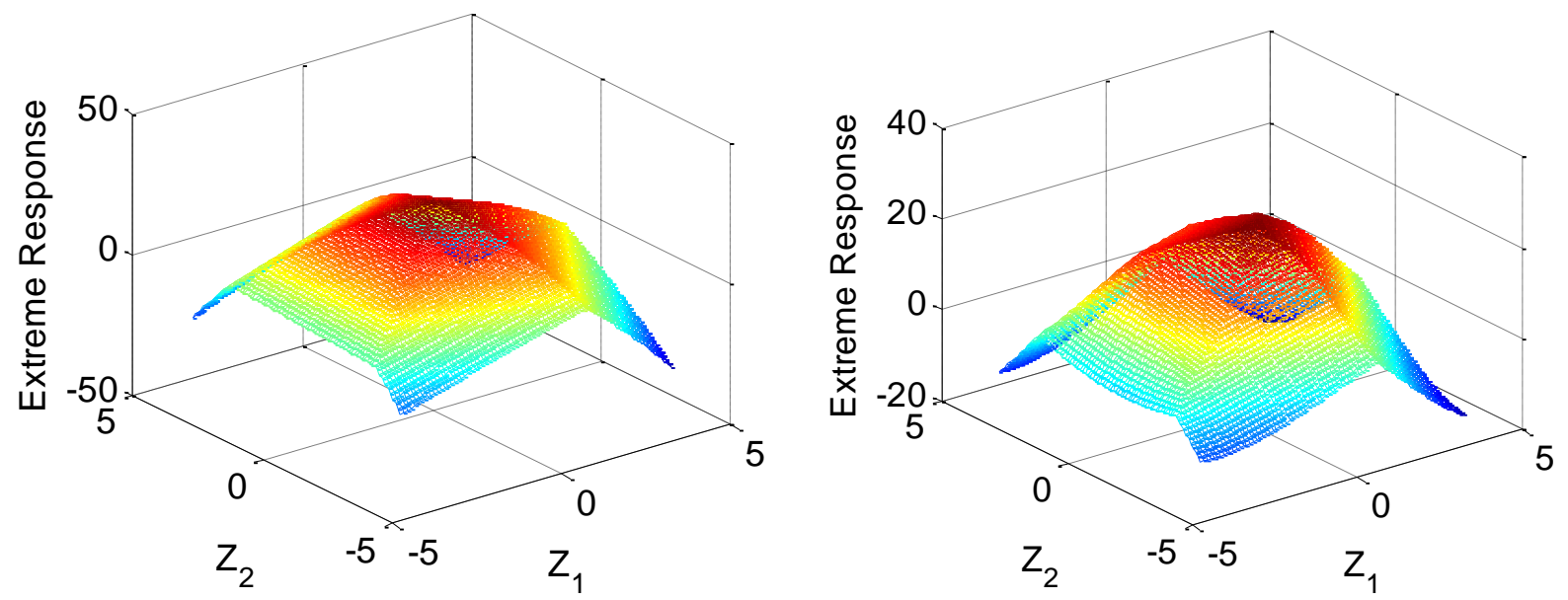

Figure 12: Extreme Response Surface by Fixing $\mathbf{X}$ a) MCS; b) AERS

The corresponding time-variant reliability is thus computed by MCS in which the response of interest is predicted by the extreme response surface. With 3660 number of function evaluations for all the initial GP models plus 1425 function evaluations for model updating, the time-variant reliability approximations can be obtained by the AERS approach with 0.99999 confidence level. The numbers of required samples in updating GP models for all time nodes are shown in Figure 13. Direct MCS with $10^{6}$ samples is also employed for the hydrokinetic turbine blade reliability analysis. The accuracy of the AERS approach is thus examined by comparing the instantaneous probability of failure and time-variant cumulative probability of failure with those from using the direct MCS method. Figure 14 a) demonstrates the approximated instantaneous probability of failure while b) shows the cumulative time-variant probability of failure. It has been observed that the AERS approach is able to track the evolution of time-variant reliability accurately. 


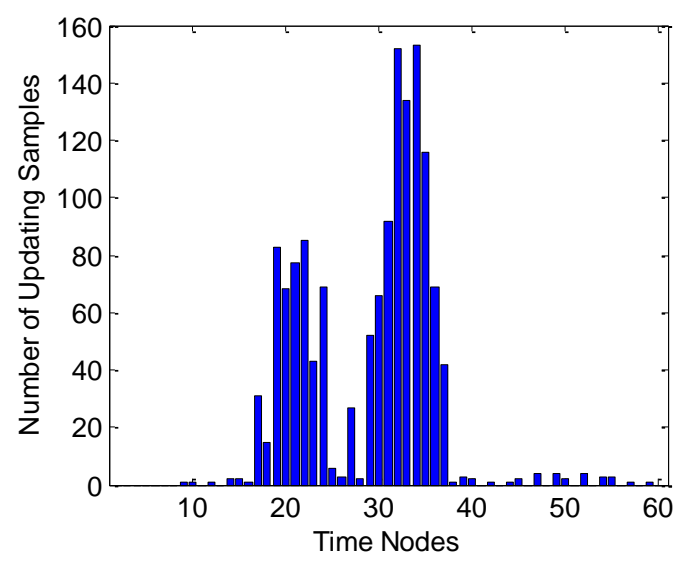

Figure 13: Number of Updating Samples

By comparing Figures 13 and 14 (a), one can observe that the number of updating samples has the same pattern with the instantaneous probability of failure. In this case, more samples points are located to the time nodes with greater probability of failure over time. It can be concluded that the AERS approach is able to identify critical time nodes for updating GP models in time-variant reliability assessment, with savings of computational cost.
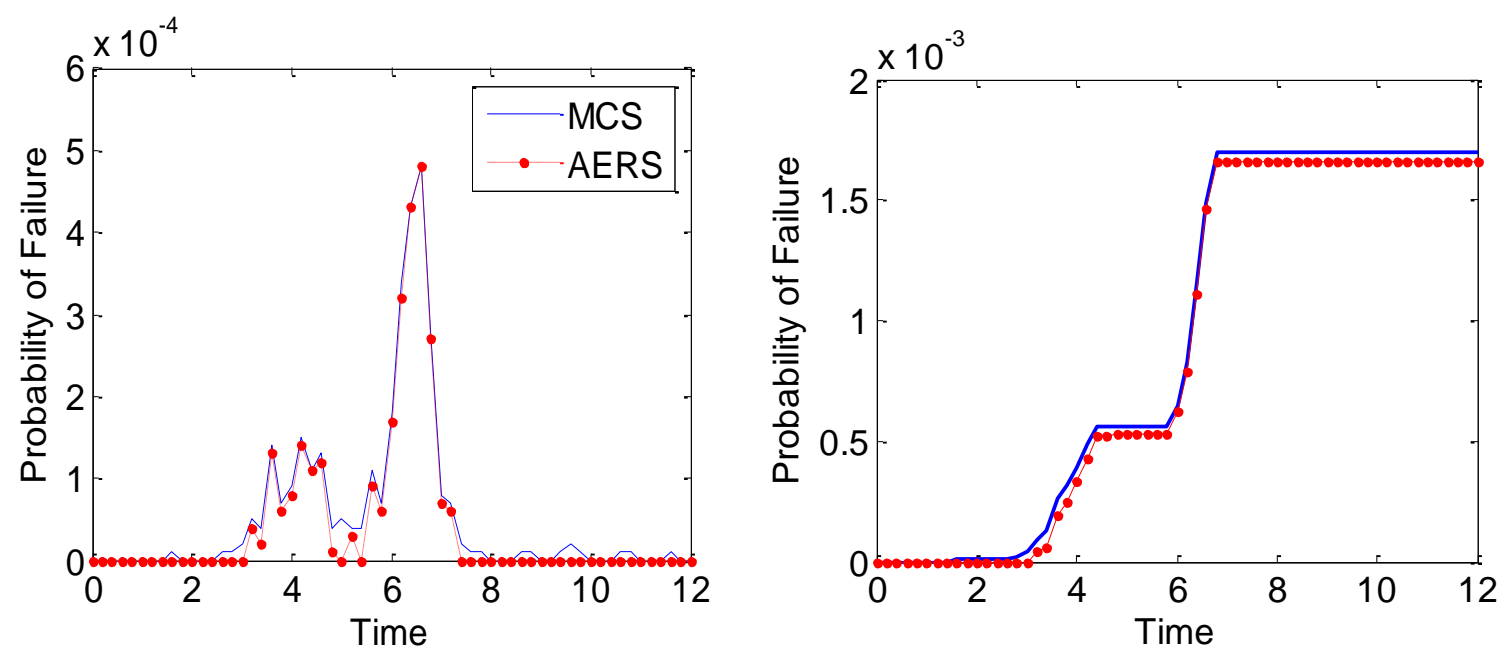

Figure 14: a) Instantaneous probability of failure; b) Time-variant Probability of Failure

\section{CONCLUSION}

This paper presents an adaptive extreme response surface (AERS) method for sensitivity-free time-variant reliability analysis with the consideration of random process parameters. The 
developed approach employs the spectral decomposition to discretize and represent random processes by a set of uncorrelated standard normal variables with the corresponding eigenfunctions. Gaussian processing (GP) surrogate models are built to predict the instantaneous limit state functions at all the discredited time nodes with uncertainty quantification. For a specific time interval, the extreme response surface is predicted by the GP models of all the time nodes within the given time interval, enabling an efficient approximation of time-variant reliability using MCS. The uncertainty of reliability approximations is quantified by a confidence level, which is used to guide the identification of critical time nodes for updating the limit state functions. A time-dependent confidence-based sampling scheme is triggered to update GP models till a confidence target is satisfied. In the GP model updating process, the confidence target should be wisely determined to balance the efficiency and accuracy of time-variant reliability assessment. In principle, a higher conference target should be assigned if a smaller probability of failure is expected. Two case studies are implemented to demonstrate the effectiveness of the AERS approach. The results show that the developed approach can substantially enhance the accuracy of time-variant reliability analysis compared with the existing analytical methods while maintaining efficiency. In addition, the developed sampling scheme is capable of identifying critical time nodes over the time interval of interest, and thus greatly enhances the efficiency.

\section{ACKNOWLEDGEMENT}

This work was performed under the following financial assistance award 70NANB14H012 from U.S. Department of Commerce, National Institute of Standards and Technology as part of 
the Center for Hierarchical Materials Design (CHiMaD).

\section{REFERENCES}

1. Youn, B.D., Choi, K. K., and Du, L., 2005, "Enriched Performance Measure Approach (PMA+) for Reliability-Based Design Optimization,” AIAA Journal, Vol. 43, No. 4, pp. 874884.

2. Youn, Byeng D., Choi, K. K., and Du, L., 2005, “Adaptive Probability Analysis Using An Enhanced Hybrid Mean Value (HMV+) Method," Journal of Structural and Multidisciplinary Optimization, Vol. 29, No. 2, pp.134-148.

3. Rahman, S. and Xu, H., 2004, "A Univariate Dimension-Reduction Method for MultiDimensional Integration in Stochastic Mechanics," Probabilistic Engineering Mechanics, Vol. 19, pp. 393-408.

4. Xu, H., and Rahman, S., 2005, "Decomposition methods for structural reliability analysis," Probabilistic Engineering Mechanics, 20(3), pp. 239-250.

5. Youn, B. D., Xi Z., and Wang, P., 2008, "Eigenvector Dimension-Reduction (EDR) Method for Sensitivity-Free Uncertainty Quantification," Struct. Multidiscipl. Optim., Vol. 37, No.1, pp13-28.

6. Paffrath, M., and Wever, U. 2007, "Adapted polynomial chaos expansion for failure detection," J Comput Phys, 226:263-281

7. Sudret, Bruno., 2008, "Global sensitivity analysis using polynomial chaos expansions." Reliability Engineering \& System Safety, 93, no. 7, 964-979.

8. Hu, C, and Youn, B.D., 2011, "Adaptive-sparse polynomial chaos expansion for reliability analysis and design of complex engineering systems." Structural and Multidisciplinary 
Optimization, 43, no. 3, 419-442.

9. Wang, Z., and Wang, P., 2013, "A Maximum Confidence Enhancement Based Sequential Sampling Scheme for Simulation-Based Design", J. Mech. Des., In Press.

10. Queipo, N.V., Haftka, R.T., Shyy, W., Goel, T., Vaidyanathan, R., and Tucker, P.K., 2005, "Surrogate-based analysis and optimization," Prog Aerosp Sci, 41(1):1-28

11. Zhuang, X., and Pan, R., 2012, "A Sequential Sampling Strategy to Improve ReliabilityBased Design Optimization with Implicit Constraint Functions,” J. Mech. Des. 134, 021002.

12. Mori, Yasuhiro, and Bruce R. Ellingwood. "Time-dependent system reliability analysis by adaptive importance sampling." Structural safety 12.1 (1993): 59-73.

13. Stewart, Mark G., and David V. Rosowsky. "Time-dependent reliability of deteriorating reinforced concrete bridge decks." Structural Safety 20.1 (1998): 91-109.

14. Wang, Zequn, and Pingfeng Wang. "A new approach for reliability analysis with timevariant performance characteristics." Reliability Engineering \& System Safety 115 (2013): $70-81$.

15. Chen, J.-B., and Li, J., 2007, "The Extreme Value Distribution and Dynamic Reliability Analysis of Nonlinear Structures with Uncertain Parameters," Struct. Safety, 29(2), pp. 77-93.

16. Wang, Z., and Wang, P., 2012, “A Nested Extreme Response Surface Approach for TimeDependent Reliability-Based Design Optimization,” J. Mech. Des. 134, 121007

17. Li, J., and Mourelatos, Z. P., 2009, "Time-Dependent Reliability Estimation for Dynamic Problems Using a Niching Genetic Algorithm," Journal of Mechanical Design, 131(7), p. 071009.

18. Mourelatos ZP, Majcher M, Pandey V, Baseski I. Time-Dependent Reliability Analysis Using the Total Probability Theorem. ASME. J. Mech. Des. 2015;137(3):031405-031405-8 
19. Lutes, L. D., and Sarkani, S., 2009, "Reliability Analysis of Systems Subject to First-Passage Failure,” NASA Technical Report No. NASA/CR-2009-215782.

20. Sudret, B., 2008, “Analytical Derivation of the Outcrossing Rate in Time-Variant Reliability Problems," Struct. Infrastruct. Eng., 4(5), pp. 353-362.

21. Hu, Z. and Du, X., 2013, "Time-Dependent Reliability Analysis with Joint Up-crossing Rates," Structural and Multidisciplinary Optimization, 2013, DOI: 10.1007/s00158-0130937-2.

22. Andrieu-Renaud, C., Sudret, B., and Lemaire, M., 2004, “The PHI2 Method: A Way to Compute Time-Variant Reliability," Reliab. Eng. Syst. Saf., 84(1), pp.75-86.

23. Rice, S. O., 1944, "Mathematical Analysis of Random Noise," Bell Syst. Tech. J., 23, pp. $282-332$.

24. Baseski, I., Drignei, D., Mourelatos, Z. P., \& Majcher, M. (2014). A New Metamodeling Approach for Time-Dependent Reliability of Dynamic Systems with Random Parameters Excited by Input Random Processes (No. 14IDM-0085--2014-01-0717). OAKLAND UNIV ROCHESTER MI DEPT OF MECHANICAL ENGINEERING.

25. Hawchar, L., El Soueidy, C. P., \& Schoefs, F. (2015, May). Time-variant reliability analysis using polynomial chaos expansion. In ICASP12 Conference.

26. $\mathrm{Hu}$, Zhen, and Xiaoping Du. "Mixed Efficient Global Optimization for Time-Dependent Reliability Analysis." Journal of Mechanical Design 137, no. 5 (2015): 051401.

27. Hu, Zhen, and Sankaran Mahadevan. "A Single-Loop Kriging Surrogate Modeling for TimeDependent Reliability Analysis." Journal of Mechanical Design 138, no. 6 (2016): 061406.

28. Drignei, D., Mourelatos, Z. P., Kosova, E., \& Baseski, I. (2015, August). Time-Dependent Reliability Using Metamodels With Transformed Random Inputs. In ASME 2015 
International Design Engineering Technical Conferences and Computers and Information in Engineering Conference (pp. V02BT03A053-V02BT03A053). American Society of Mechanical Engineers.

29. Jones, D. R., Schonlau, M., \& Welch, W. J. (1998). Efficient global optimization of expensive black-box functions. Journal of Global optimization, 13(4), 455-492.

30. Lee, T. H., and Jung, J. J., 2008, "A Sampling Technique Enhancing Accuracy and Efficiency of Metamodel-Based Rbdo: Constraint Boundary Sampling," Computers \& Structures, 86(13), pp. 1463-1476.

31. Echard, B., Gayton, N., and Lemairem, M., "AK-MCS: an active learning reliability method combining Kriging and Monte Carlo simulation." Structural Safety 33.2 (2011): 145-154.

32. Chen, Z., Peng, S., Li, X., Qiu, H., Xiong, H., Gao, L., \& Li, P. (2015). An important boundary sampling method for reliability-based design optimization using kriging model. Structural and Multidisciplinary Optimization, 52(1), 55-70.

33. Quinonero-Candela, J., Rasmussen, C.E., and Williams, C., 2006, "Approximation methods for Gaussian process regression," In: Large-scale kernel machines adaptive computation and machine learning. The MIT Press, Cambridge, MA.

34. Lloyd, C. D., and Atkinson, P.M., 2001, "Assessing uncertainty in estimates with ordinary and indicator Kriging," Computers \& Geosciences, 27, no. 8, pp.929-937.

35. Simpson, T.W., Mauery, T.M., Korte, J.J., and Mistree, F., 2001, "Kriging models for global approximation in simulation-based multidisciplinary design optimization," AIAA Journal, 39, no. 12 , pp.2233-2241.

36. Kleijnen, J.P.C., 2009, "Kriging metamodeling in simulation: a review," European Journal of Operational Research, 192, no. 3, pp.707-716. 
37. Jones D.R., Schonlau, M., Welch, W.J., 1998, "Efficient global optimization of expensive black-box functions," Journal of Global Optimization, 13: 455-492.

38. Hu, Z., and $\mathrm{Du}, \mathrm{X} ., 2012$, "Reliability Analysis for Hydrokinetic Turbine Blades," Renewable Energy, 48, pp. 251-262 31005-1, 2011. 